\title{
Transport and Installation of the Dark Energy Survey CCD Imager
}

\author{
Greg Derylo ${ }^{1}$, Edward Chi, H. Thomas Diehl, Juan Estrada, Brenna Flaugher, Ken Schultz \\ Fermi National Accelerator Laboratory, Batavia, Illinois, USA 60510
}

\begin{abstract}
The Dark Energy Survey CCD imager was constructed at the Fermi National Accelerator Laboratory and delivered to the Cerro Tololo Inter-American Observatory in Chile for installation onto the Blanco 4m telescope. Several efforts are described relating to preparation of the instrument for transport, development and testing of a shipping crate designed to minimize transportation loads transmitted to the camera, and inspection of the imager upon arrival at the observatory. Transportation loads were monitored and are described. For installation of the imager at the telescope prime focus, where it mates with its previously-installed optical corrector, specialized tooling was developed to safely lift, support, and position the vessel. The installation and removal processes were tested on the Telescope Simulator mockup at FNAL, thus minimizing technical and schedule risk for the work performed at CTIO. Final installation of the imager is scheduled for August 2012.
\end{abstract}

Keywords: Imager, transport, Blanco, CTIO

\section{INTRODUCTION}

The Dark Energy Survey (DES) ${ }^{1}$ built a CCD imager for the 4 meter Blanco telescope at the Cerro-Tololo International Observatory (CTIO). This 2.2 sq. deg. camera utilizes 62 2k x 4k 0.250mm-thick CCDs and 12 2k x 2k CCDs for guiding and focus control, totaling 0.57 Gpixels. Fabrication, assembly, and testing of the imager was performed at the Fermi National Accelerator Laboratory (FNAL) near Chicago ${ }^{2}$. Shown in Figure 1, the imager consists of a vacuum vessel around which are mounted several electronics crates and vacuum pumps and configured for shipping it weighs nearly $600 \mathrm{~kg}$.

The imager represents a considerable investment in capital and time and numerous factors were taken into consideration to minimize shipping-related risks. The first issue to be considered was whether or not the imager was to be shipped fully assembled. Considering the speed with which the project team could conceivably depopulate and repopulate CCD modules on the focal plane, it was feasible to remove all 74 CCD modules and ship them in their individual storage boxes. With the CCDs removed, the readout crates could also be removed. Shipment of the remaining hardware would have been very straightforward with greatly minimized risk of damage. However, the imager assembly and testing was done in stages, with only a fraction of the CCDs installed in each round, and it was felt that this same approach should be carried out for such a reassembly-after-shipping scenario. With each assembly/testing stage requiring a few weeks of work, it was decided that shipping the fully-populated imager was necessary in order to avoid having to repeat that staged approach. In order to safely ship the imager and its delicate contents, it was therefore necessary to develop a special shipping crate to cushion the fully-assembled imager during transport. The development of this crate and a discussion of various elements of the transport experience are included in Section 2 below.

The final assembly step for the imager is to mount it at the Blanco prime focus, attaching it to the previously-installed corrector that houses the first four elements of the camera optics. This installation step is scheduled for August of 2012 but is a process already practiced on the Telescope Simulator built at FNAL ${ }^{3}$. Section 3 describes the installation process and the development of the tooling used. Having already tested the installation equipment and processes on the

${ }^{1}$ Derylo@fnal.gov, 1-630-840-6104 
simulator, the technical and schedule risks have been minimized and it is hoped that the installation work will proceed smoothly at CTIO.
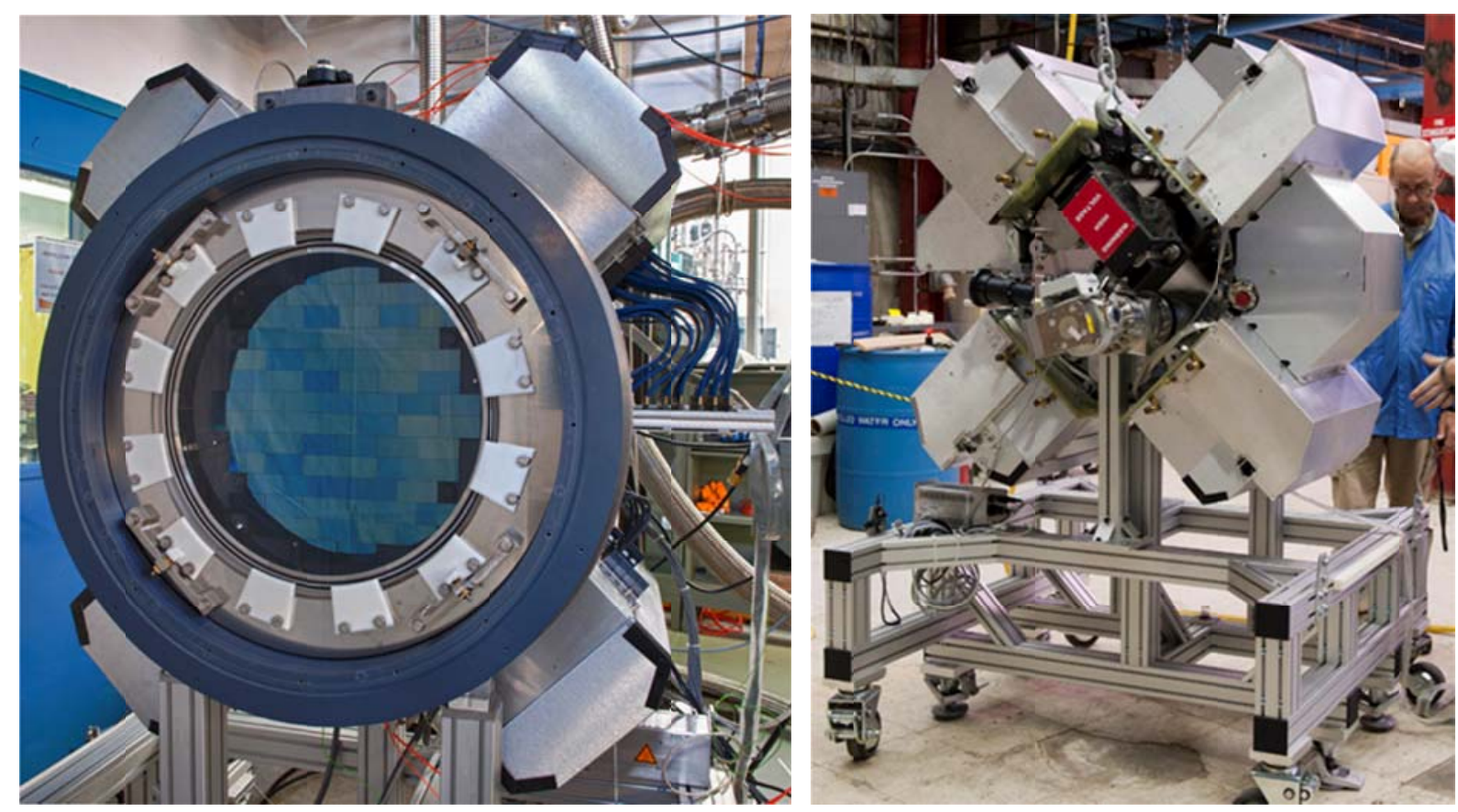

Figure 1. The DES imager front and rear views. The thick grey ring around the window is used to support bolt-on testing equipment or a protective lens cover.

\section{TRANSPORT FROM FNAL TO CTIO}

\subsection{Development of the Imager Shipping Crate}

In order to plan for the safe transport of such an instrument, the magnitude of the allowable loads transmitted to the imager must first be defined. Such a limit was not arrived at analytically, and it was certainly not to be determined by testing. Rather, an allowable limit was arrived at based on previous handling experience. During assembly at FNAL, the imager was routinely transported on its handling cart (Figure 1) between the cleanroom, where all vessel internal work was done, and the testing area in the adjacent building, where it was connected to the readout electronics and liquid nitrogen plumbing system. A shock logger (Sensr model GP1 ${ }^{4}$ ) mounted on the cart indicated maximum accelerations between 3 and 4 G's during these handling operations. Based on that experience it was expected that the imager could safely withstand shock loads of 3 G's during shipping.

Expected transportation loads by truck and aircraft have been reported to be approximately 6 G's vertically and 3.5 G's horizontally ${ }^{5}$. However, this fails to capture handling loads during shipment transitions (i.e., forklift loads). Transport of the NEWFIRM instrument from Arizona to CTIO found that the maximum load of about 6 G's was applied during such handling ${ }^{6}$.

Since the allowable load on the payload is so much smaller than that expected during transport, a specialized shipping container that provides a large amount of cushioning was needed. A rigid mounting cradle was designed to support the imager during transport and the shipping crate was to interface with the base of this cradle. It was desired to contract crate design and fabrication with a specialty shipping company and a specification was written to define the project needs. The contract was to include validation testing at an independent testing lab using the support cradle and a FNALsupplied dummy weight to simulate the imager weight and center of mass. The contract was awarded to Nefab Chick Packaging $^{7}$ who designed a crate using wire rope vibration isolators to cushion the payload. These isolators reduce 
shock loads by transmitting input energy more slowly, which results in larger motions spread out over longer periods but with a reduction in maximum pulse heights. The crate, with cradle and mockup weight, is shown in Figure 2.
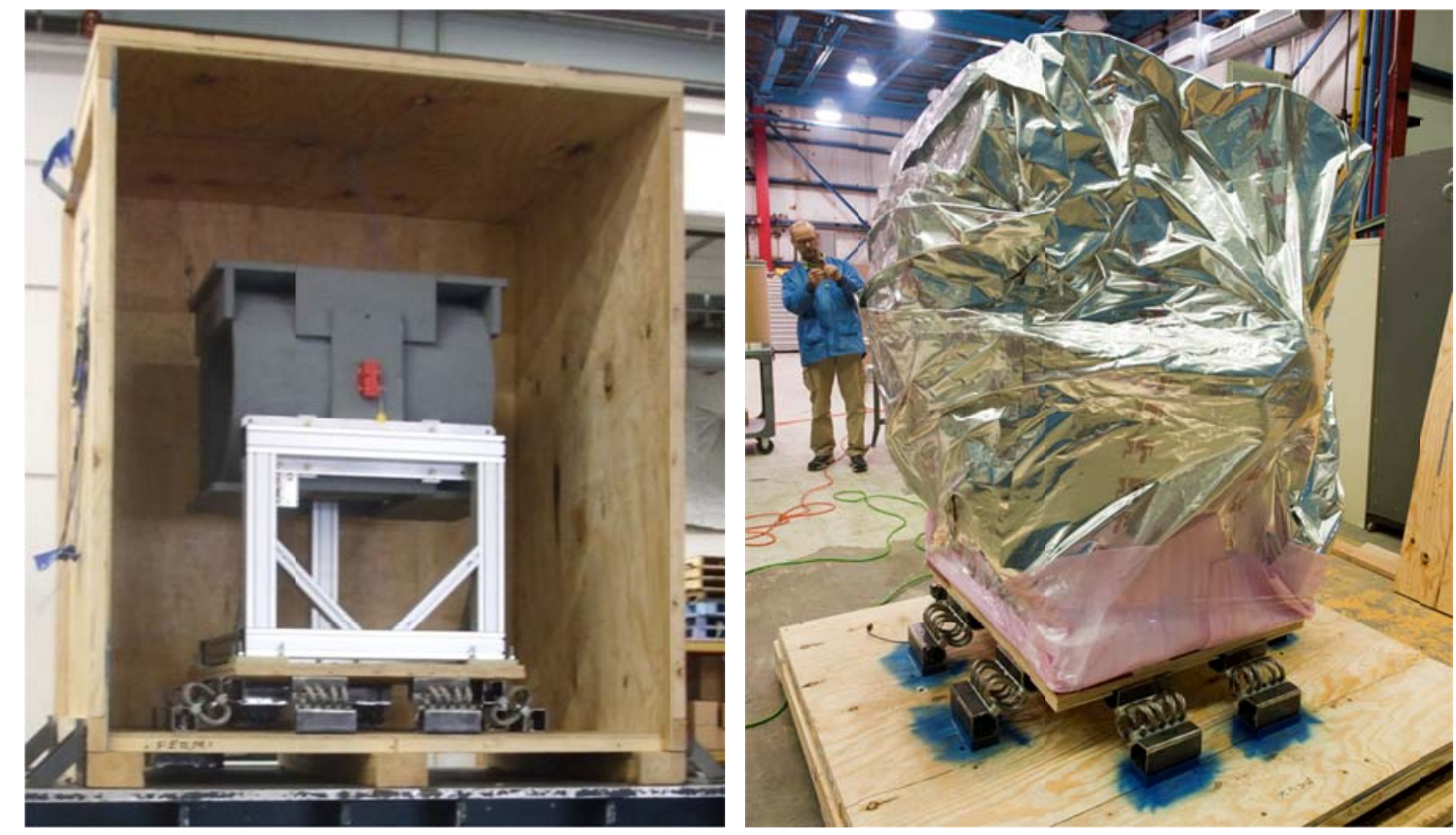

Figure 2. The shipping crate internals showing the vibration isolation coils supporting the imager payload. The photo on the left shows crate testing at an independent testing laboratory using a mockup weight. The photo on the right shows the DES imager being prepared for shipping to CTIO, where the imager is wrapped in ESD-safe foam sheeting and a vapor barrier.

The crate's performance was validated during a day of testing at Pira International ${ }^{8}$ and consisted of several batteries of tests. Horizontal shock impact testing was performed on all four sides at several speeds up to $1.2 \mathrm{~m} / \mathrm{s}$, corresponding to approximately 15 G's. Accelerometer data from the payload indicated transmitted shocks of less than 1.1 G's. Vertical shocks were applied by first lifting one edge of the crate and supporting it on a spacer block up to $10 \mathrm{~cm}$ thick. The spacer block was then rapidly pulled out, inputting shocks to the crate base in excess of $30 \mathrm{G}$ 's. For these cases, the maximum transmitted shock load was just under 3 G's. The wire rope vibration isolators therefore did an impressive job of shock isolation. However, a problem was identified with the crate design when the horizontal testing was repeated with one wall of the crate removed. The payload motion was found to be so large that if the real imager had been in place rather than the smaller mockup weight, its various protrusions could have struck the wall of the crate. Therefore, the shell of the crate was remade by Nefab to provide additional internal clearance.

Validation testing continued with vertical vibration. A frequency sweep up to $200 \mathrm{~Hz}$ found an isolation coil natural frequency at about $4 \mathrm{~Hz}$ with a peak transmissibility of 2.2. This frequency is just outside the anticipated 2 to $3 \mathrm{~Hz}$ resonant frequency of an air ride truck suspension, which was a cause for some concern. Another design problem was identified during the vertical vibration test that was discovered as a result of observations again made with one side of the crate removed. Large payload motions resulted in contact between the wire rope coil and the floor of the crate. This resulted in increased loads transmitted to the payload and could be seen as sudden spikes in the mockup-weight-mounted accelerometer data. This issue was addressed with the addition of spacer plates added underneath the isolation coil mounting brackets for the final version of the crate.

Further vibration table testing was then performed with air-ride-truck simulation programs of various intensity levels. Accelerometer response for the simulation of a typical ride is shown in Figure 3 and indicates that vibration energy applied near the natural frequency does indeed result in a heightened response at this region. Having a low natural frequency is a direct outcome of having good shock isolation properties, but the magnitude of the amplification factor is 
limited due to the internal damping of the isolation coils resulting from internal friction between individual wire strands. Given the good shock isolation response, which was of primary importance, the relatively mild amount of amplification, and the improvement anticipated with the addition of the mounting bracket spacer plates, this frequency response behavior was considered to be acceptable.

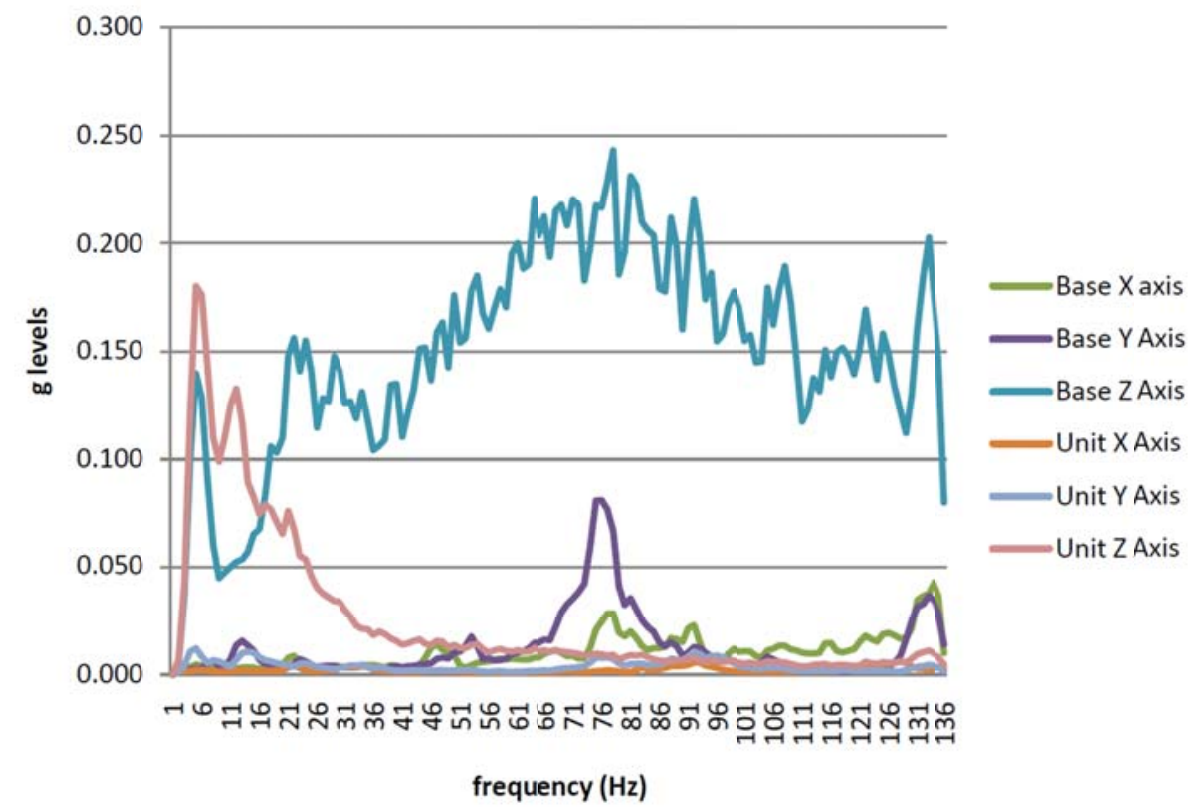

Figure 3. Vibration test spectral response of the shipping crate to a simulated air-ride-truck trip. The "Base" accelerometer is mounted to the crate floor while the "Unit" instrument is mounted to the imager mockup weight.

\subsection{Preparing the Imager for Transport}

Several actions were taken to remove transportation risks. The most visible one was to replace the front window and its mounting cell with a blank cover. The window cell, with protective covers over both faces, was shipped in a separate padded crate. This was not the powered window lens, but rather a flat window used during imager testing. The real window and its cell were shipped separately along with the full set of corrector optics.

The cables connecting the readout crates to the vacuum interface board were also removed for safety and shorting plugs were inserted in all the CCD connectors on the interface board for electrical protection. With the cables removed it would have been preferable to then also remove the electronics readout crates and ship them separately, but the cable removal decision was made too late in the planning process for removing the crates would have changed the weight of the payload by $15 \%$ and therefore altered the behavior of the vibration isolation coils. So the electronics crates were left in place.

The double-walled liquid nitrogen lines that cantilever significantly outward from the imager vessel were also removed to reduce shipping risk. Temporary brackets were then added to the liquid nitrogen piping inside the vessel in order to constrain it in place after its supporting feedlines had been removed. Finally, temporary brackets were added to the back side of the focal plane support plate to which all the CCD modules were mounted. Braces that extend outwards from these brackets prevented lateral movement of the plate on its thin bipod legs. No constrains were added to reinforce this plate in the imager's axial direction since the bipods are extremely stiff in that direction.

The imager was shipped under vacuum, primarily for the benefit of the ion pump mounted to the imager vessel.

\subsection{Shipping the Imager}

At FNAL the imager vessel was transferred into its cradle in the shipping crate. Staff from the shipping crate vendor were on hand on packing day to help wrap the camera in ESD-safe foam and a heat-sealable vapor barrier (Figure 2). A sealed port had been prepared for the vapor barrier to allow pass-through of a cable for the shock logger. The two Sensr 
GP1 loggers ${ }^{4}$, one on the crate base and the other on the imager vessel itself, were connected to a patch panel added to one wall so they could be read out with a laptop from outside the crate.

Transport of the imager crate, as well as several other crates of less-sensitive support equipment, was contracted with an international shipping company ${ }^{9}$. The load was first moved by air-ride truck from the Chicago area to the airport in Miami. This was by dedicated truck, so no additional stops or load rearranging was necessary en route, thus minimizing shipping risk. After flying from Miami to Santiago, it was trucked from the airport to the mountaintop.

Figure 4 shows the $\mathrm{X}, \mathrm{Y}$ and $\mathrm{Z}$ axis shock logger data for the move. The loggers saved the minimum and maximum values over 1-minute intervals for the entire move. The blue curves show the minimum and maximum data for the logger mounted to the crate base. The red curves with small dots show the response of the imager-mounted logger. The data shows the three main periods of the move. The initial 'hashy' portions of the data represent the truck ride to Miami on November $17^{\text {th }}$ and $18^{\text {th }}$. The vertical-direction data shows that some of the imager loads are larger than the loads applied to the crate base, indicating that there was some excitation at the isolation coil resonant frequency mentioned above. However, at no point did this result in a dynamic load larger than 2 G's.

The middle portion of the plot shows handling in Miami, the Miami-to-Santiago flight on November $20^{\text {th }}$ and $21^{\text {st }}$, and handling in Santiago. Handling in Miami resulted in the highest load applied to the crate: 4.9 G's. However, the isolation coils limited the resulting load on the imager to $2.1 \mathrm{G}$ 's.

The final portion of the plot on November $22^{\text {nd }}$ and $23^{\text {rd }}$ shows the drive from Santiago to the telescope, with an additional small response associated with craning of the imager to the main floor of the telescope dome. This ride has an overnight break in it since the last portion of the drive, up the observatory's gravel road to the mountaintop, was delayed until the next morning rather than doing it that evening.

\subsection{Unpacking of the Imager and Preparing it for Final Testing}

After first checking the shock logger data to verify that the loads on the imager were within acceptable levels, the crate was opened and a tilt in the way the imager sat in the crate was noticed. Examination found what appears to be some loosening of the vibration isolation coil itself along one edge of the suspension system, as shown in Figure 5, and the surface of the crate floor shows some sign of coil contact here. This degradation is possibly due to extended operation near the natural frequency. Although the imager was delivered safely, in light of this observation it may have been desirable to trade some reduction in shock isolation capability for increased stiffness and therefore more separation from the expected natural frequency of the truck transportation.

The imager was then transferred back onto its handling cart and moved into the cleanroom facility in the Blanco dome. There it was opened and the transportation-related hardware changes described above were reversed. While the front cover of the vessel was off, a quick flatness scan of the CCD surfaces was done to ensure that the spring-mounted CCDs were still solidly seated against the focal plane support plate. The Micro-Epsilon confocal chromatic displacement sensor was mounted on a simple rail system, shown in Figure 6. This rail system was not intended to be used to do an accurate survey of the entire array, but rather just perform a quick check of a CCD relative to its immediate neighbors. The same instrument, mounted to a more accurate positioning system, is used to perform more accurate scanning of the array through the flat window under both warm and cold conditions ${ }^{2}$. After reassembly, the imager was ready for electrical and plumbing connections and the beginning of post-transport validation testing. 

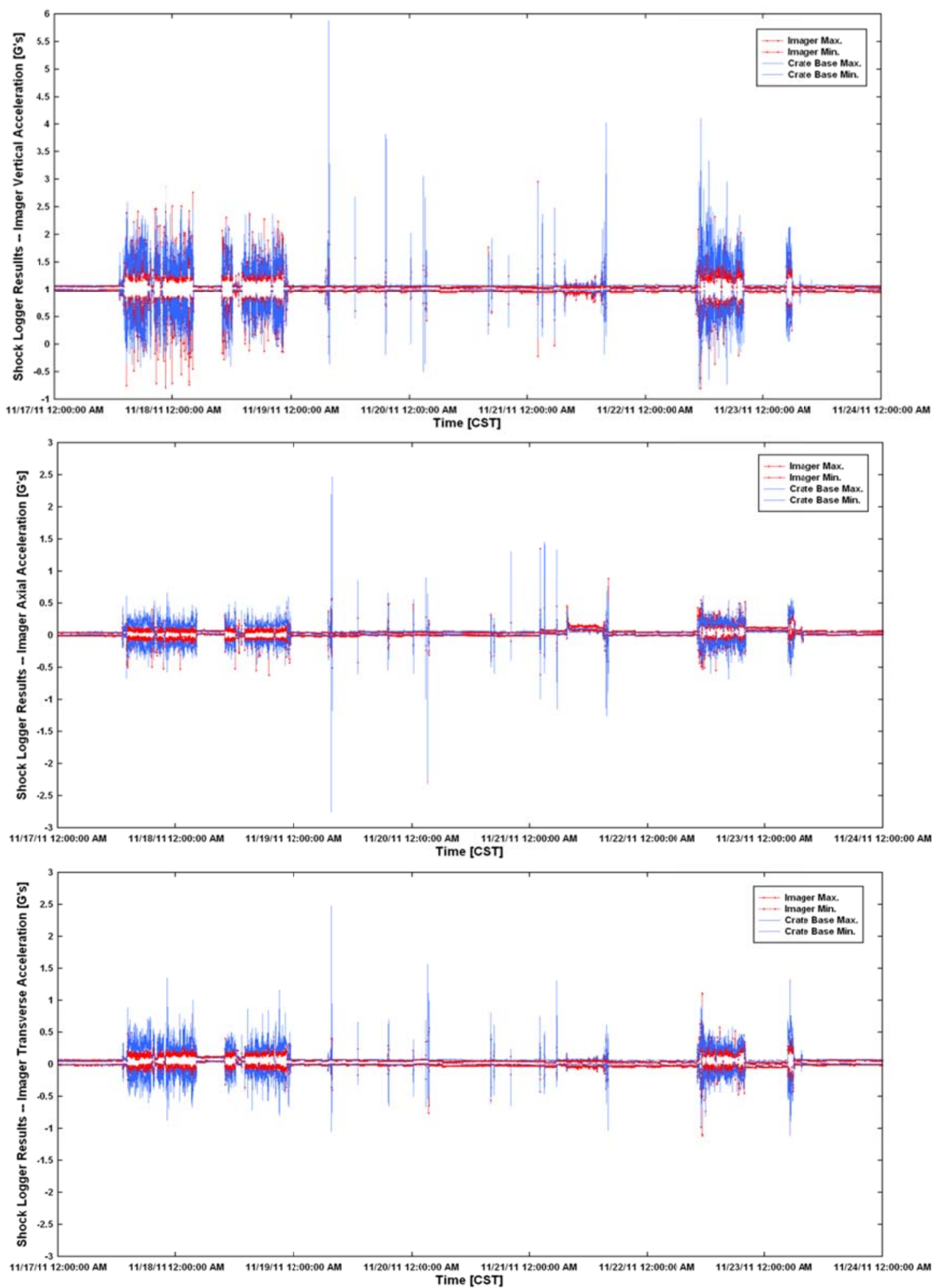

Figure 4. Transport shock logger results in the vertical, axial, and transverse directions. Blue data shows the minimum and maximum shock loads experienced by the crate base. Red data shown with small data points represents the minimum and maximum accelerations for the imager payload. 


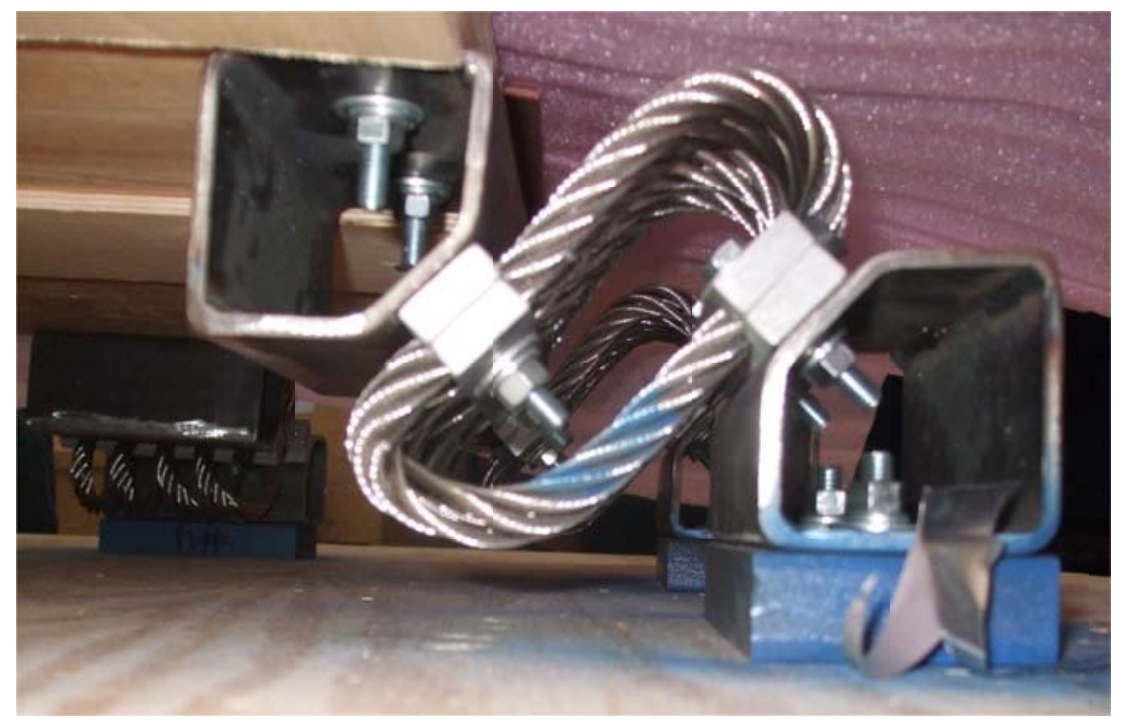

Figure 5. Isolation coils after arrival in Chile. View along listing edge of the suspension system with the imager not yet removed from crate. Crate floor shows some sign of coil contact even with the additional spacer shown under the coil mounting bracket.

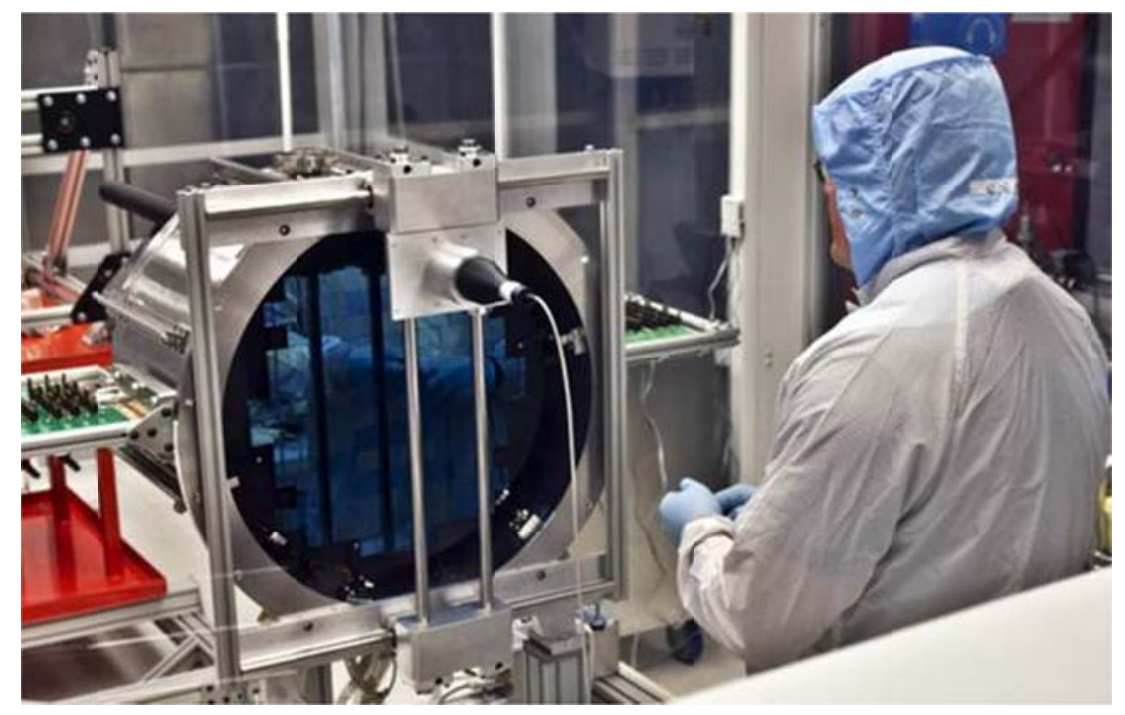

Figure 7. The Micro-Epsilon confocal chromatic displacement system being used to perform a quick scan of the CCD array after arrival at the Blanco telescope. The simple rail system was built to allow quick inspection of a CCD surface relative to its immediate neighbors. The same instrument, mounted on a precision stage system, is separately used to survey the focal plane array through the flat vessel window. Photo credit T. Abbott (CTIO). 


\section{IMAGER INSTALLATION ON THE TELESCOPE}

\subsection{Telescope Preparation}

The imager vessel is the last major component of the DES instrument to be installed. With the corrector barrel on its hexapod positioning system already mounted at prime focus, the telescope is to be moved to its maintenance station $7.5^{\circ}$ above the horizon and secured in place. There the imager is to be installed through the opening in the end of the prime focus cage and mounted to the corrector.

\subsection{Handling the Imager}

With the perimeter of the imager vessel largely covered with readout electronics and cooling connections, only selected points on the back face of the vessel are available for supporting the weight of the imager. The supports must be long enough to reach past the services at the end of the cage, through the cage opening, and past the vacuum pumps mounted on the rear of the imager vessel and still provide enough stiffness to rigidly support the imager's $640 \mathrm{~kg}$ weight. This was done with a set of six rods that screwed into brackets mounted onto the back of the imager vessel, as shown in Figure 8. A ring inserted over the ends of the rods is held in place with shaft collars and serves two important functions: it stiffens the rod assembly by constraining them all together, and hoist rings added to it serve as a pickup point out past the electronics crates that surround the vessel. During rigging operations, an additional pickup point is used at the front of the vessel by use of a temporary lifting bracket added to the vacuum window flange as shown in Figure 9.

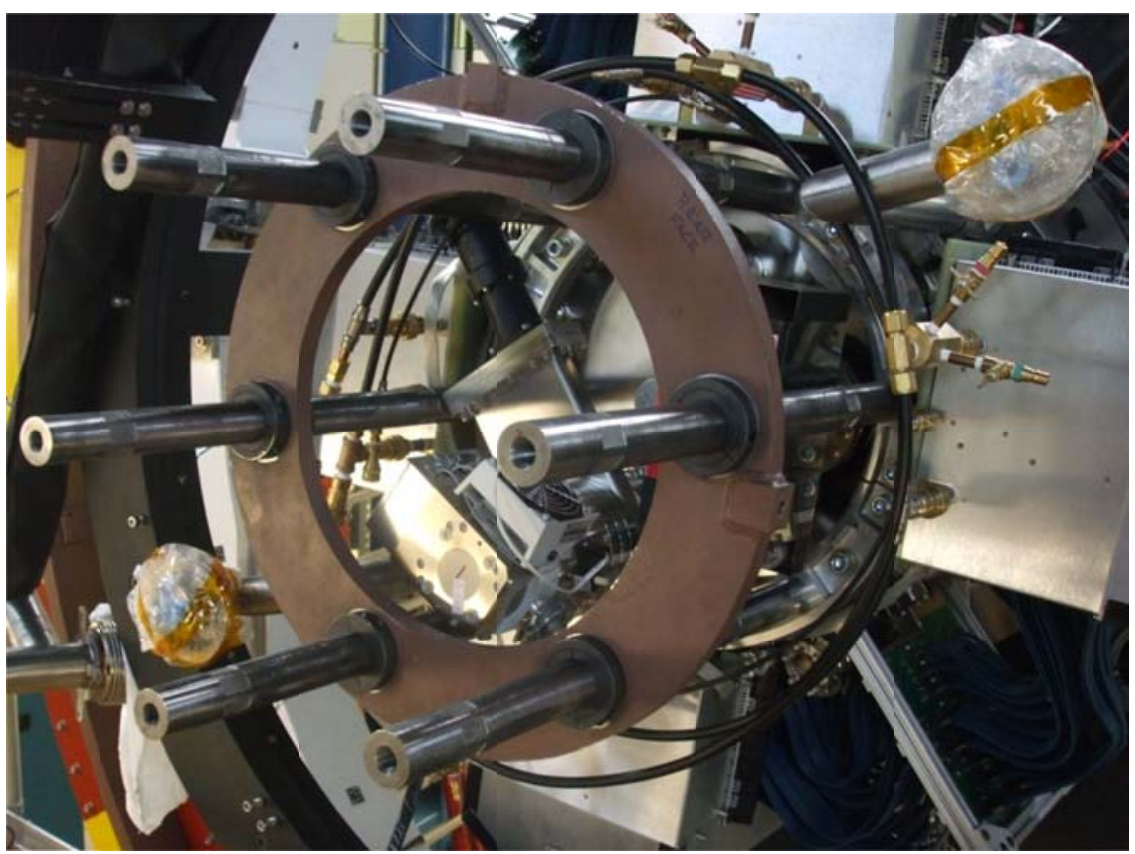

Figure 8. Set of six handling rods threaded into brackets on the rear of the imager vessel. The ring increases stiffness of the rod assembly and acts at a lifting point when moving the imager by crane. The two liquid nitrogen plumbing flanges are shown covered with plastic wrapping for handling protection.

\subsection{Installing the Imager}

At the Northwest platform maintenance station, the installation tooling makes use of selected existing hardware used to position equipment there. The rods extending from the rear of the imager attach to a set of plates mounted to the movable slide (Figure 9). The slide hardware allows adjustment of transverse position and tilt angle as it moves axially along its length. The set of plates attached to the front of the slide mount are designed to provide adjustment in vertical position and roll angle. All the motions are necessary to move the imager through the opening in the end of the prime focus cage, where there is approximately $19 \mathrm{~mm}$ of radial clearance, and onto the rear of the corrector (Figure 10), where the mounting bolts and the expandable/removable locating pins ${ }^{10}$ can be installed (pins are removed after imager mounting to preserve electrical isolation between the imager and the corrector). The installation fixture motion adjustments therefore cover five out of the six motion types; what is missing is yaw angle control, so careful initial setup work will be necessary to ensure that the fixture equipment is well-aligned to the telescope for this operation. This 
alignment will be confirmed prior to imager installation when this same fixture is used to remove the imager mockup weight currently mounted to the DES corrector.

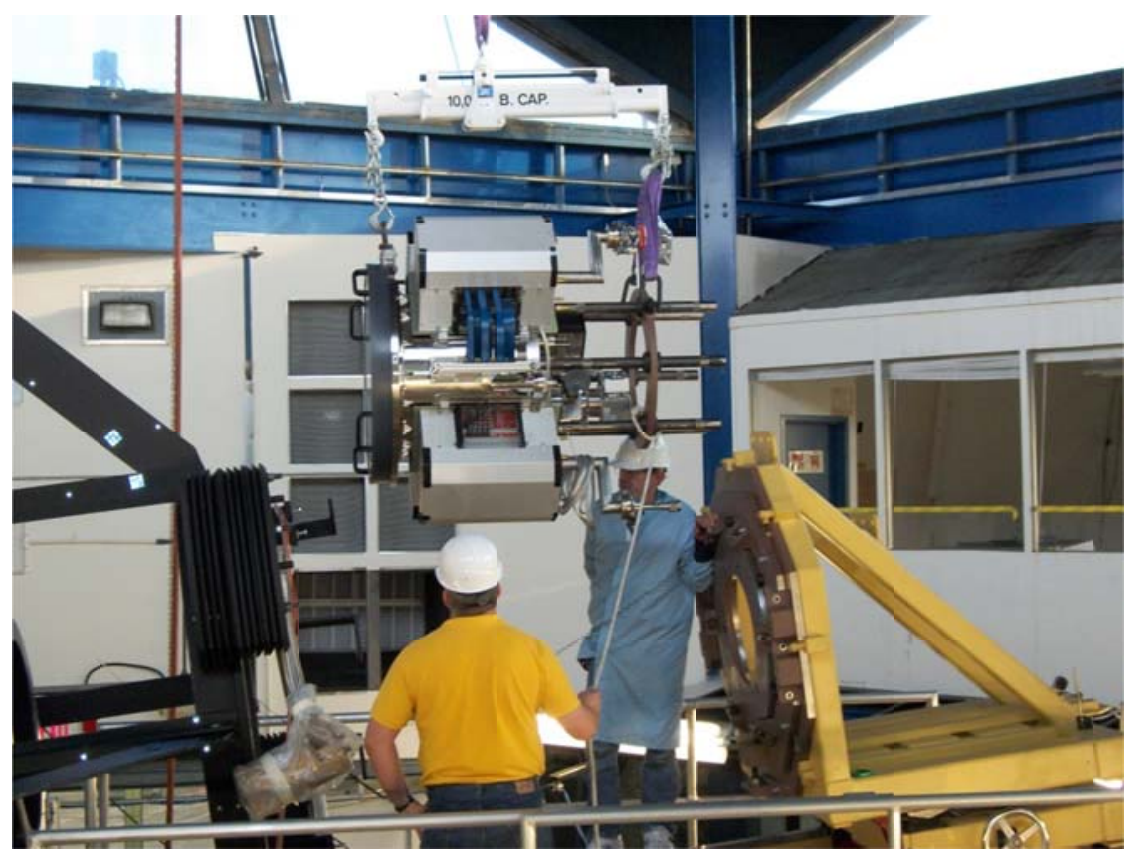

Figure 9. Imager installation testing on the Telescope Simulator. The crane is being used to transfer the imager onto its installation fixture. The fixture supports the cantilevered imager and permits adjustment of its transverse \& vertical position and its pitch \& roll angles as it moves axially into the prime focus cage where it mates it with the corrector.

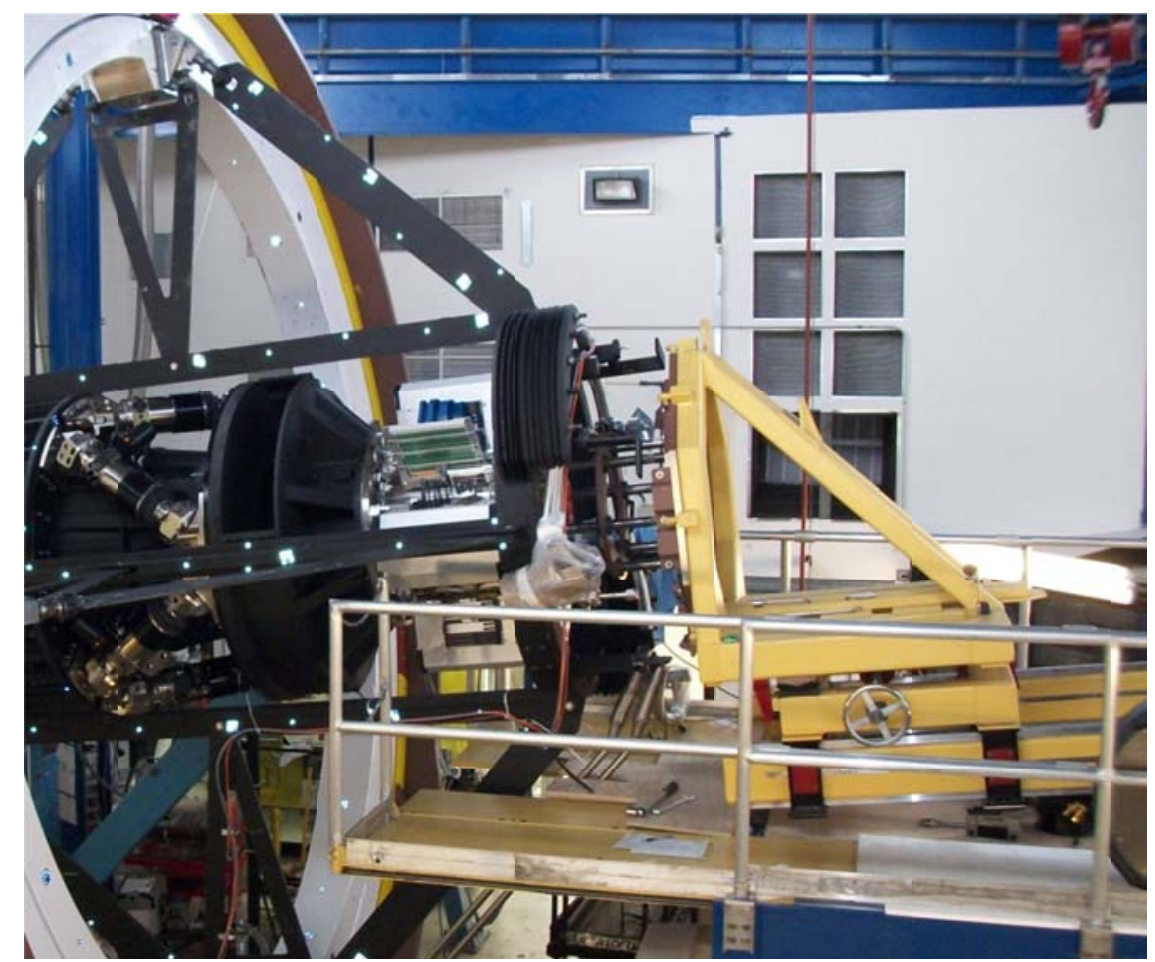

Figure 10. Imager installation testing on the Telescope Simulator. The imager has been translated forward and mated to the back of the corrector. 
As shown in Figures 8 through 10, the installation process was tested successfully on the Telescope Simulator built at FNAL. Having completed the process beforehand significantly reduces the technical and schedule risks associated with such a critical handling step to be performed at a remote worksite. Installation of the imager on the Blanco telescope is scheduled for August of 2012.

\section{CONCLUSION}

A special transportation crate was developed for shipment of the fully-assembled DES imager from FNAL to CTIO, and lessons learned during validation testing with a mockup payload played an important role in achieving transport success. During shipping, transportation loads were within expected levels but there were signs of some excitation at the crate suspension system's natural frequency. However, the crate damping still provided adequate cushioning to the imager, which experienced loads less than the specified allowable level and which arrived safely at its new home.

Specialized tooling has been developed for handling the imager and installing it on the corrector in the prime focus cage. The opportunity to test the installation procedure and equipment on the Telescope Simulator at FNAL was an extremely valuable confirmation of the installation plans and helped to minimize technical and schedule risk.

\section{ACKNOWLEDGEMENTS}

Funding for the DES Projects has been provided by the U.S. Department of Energy, the U.S. National Science Foundation, the Ministry of Science and Education of Spain, the Science and Technology Facilities Council of the United Kingdom, the Higher Education Funding Council for England, the National Center for Supercomputing Applications at the University of Illinois at Urbana-Champaign, the Kavli Institute of Cosmological Physics at the University of Chicago, Financiadora de Estudos e Projetos, Fundação Carlos Chagas Filho de Amparo à Pesquisa do Estado do Rio de Janeiro, Conselho Nacional de Desenvolvimento Científico e Tecnológico and the Ministério da Ciência e Tecnologia, the Deutsche Forschungsgemeinschaft and the Collaborating Institutions in the Dark Energy Survey.

The Collaborating Institutions are Argonne National Laboratories, the University of California at Santa Cruz, the University of Cambridge, Centro de Investigaciones Energeticas, Medioambientales y Tecnologicas-Madrid, the University of Chicago, University College London, DES-Brazil, Fermilab, the University of Edinburgh, the University of Illinois at Urbana-Champaign, the Institut de Ciencies de l'Espai (IEEC/CSIC), the Institut de Fisica d'Altes Energies, the Lawrence Berkeley National Laboratory, the Ludwig-Maximilians Universität and the associated Excellence Cluster Universe, the University of Michigan, the National Optical Astronomy Observatory, the University of Nottingham, the Ohio State University, the University of Pennsylvania, the University of Portsmouth, SLAC, Stanford University, the University of Sussex, and Texas A\&M University.

\section{REFERENCES}

[1] Flaugher, B. et al., "Status of the Dark Energy Survey Camera (DECam) Project," in these proceedings.

[2] Derylo, G. et al., “Assembly of the Dark Energy Survey CCD Imager,” Proc. SPIE, Volume 7739 (2010).

[3] Diehl, H.T. et al., “Testing the Dark Energy Camera on a Telescope Simulator,” Proc. SPIE, Volume 7735 (2010).

[4] SENSR Co., Elkader, Iowa, USA. http://www.sensr.com

[5] NASA SP-8077, “Transportation and Handling Loads,” 1971.

[6] Probst, R. et al., “There and Back Again: Sharing a Major Instrument Between Hemispheres,” Proc. SPIE, Volume 7735 (2010).

[7] Nefab USA, Elk Grove Village, Illinois. http://www.nefab.us 
[8] Pira International Lansing Technical Service Center, Lansing, Michigan, USA. http://www.smitherspira.com

[9] DHL Worldwide Express. http://www.dhl.com

[10] S.B. Whistler \& Sons Inc., Medina, New York, USA, http://www.sbwhistler.com/met.htm 